\title{
Visual Babble Polyglot / Babil visuel polyglotte
}

\section{Gabrielle Bonifaci, with TJ Charlton}

Video 1'50 with sound, white on black

April 2013

This piece involves several dissolutions.

Solid language - tactile, as in braille or through touching - is evoked in written languages that can be read and scrutinized, but not touched.

In turn, the variety of these - untranslated and untranslatable - languages dissolves meaning(s) into innumerable shards of cultural and linguistic twists. More than thirty languages from around the world, without counting variations and dialects, are represented. Understanding will be limited to each reader's individual language skills and multicultural appetence. The rest will be a guessing game and, more so, something unique and inaccessible.

Worse, a few of these languages are in danger of extinction and could soon vanish from our collective knowledge.

Another dissolution results from the iridescence of literary writing, its images, reflections and shadows, its many constellations. Meanings thus multiply and, in turn, desegregate in everwidening circles and riffs.

Yet our dissolutions go further.

The paper on which words were inscribed, was cut with scissors, scanned, saved, edited, modified, digitalized. In the process, the paper disappeared.

The words made images were then layered, superimposed, blurred or melted into one another until they became so elusive that we could no longer distinguish them. The resulting video is meant to perform a fluid gesture of erasure.

Finally, the accompanying sound effects - Ominous dance floor, Circuit dialog, Gorai atmosphere, Samoa sweep, and Serena swirl - were chosen because they countered such pliancy. Intending to avoid any impression of spirituality, we selected cavernous sounds that disrupted and silenced the visual/linguistic flow. Our words are now engulfed into the abyss of these sunken accents - their final dissolution.

\section{Babil visuel polyglotte / Visual Babble Polyglot}

\section{Gabrielle Bonifaci, en collaboration avec TJ Charlton}

Vidéo 1'50 avec son, blanc sur noir

Avril 2013

Ce travail associe plusieurs dissolutions.

Le langage solide, c'est-à-dire tactile, qu'il s'agisse de braille ou du toucher, est évoqué dans des langues écrites qui peuvent être lues et scrutées, mais pas touchées.

A son tour, la variété de ces langues - sans traductions et intraduisibles - dissout le(s) sens en d'innombrables éclats de rebondissements linguistiques. Plus de trente langues du monde entier, sans compter leurs variations et dialectes, sont représentées. Néanmoins, leur compréhension est limitée par les compétences linguistiques et l'appétence multiculturelle de chaque lecteur. Le reste restera un jeu de devinettes et, plus encore, quelque chose d'unique et d'inaccessible.

Pire, certaines de ces langues sont en danger d'extinction et pourraient bientôt disparaître de notre savoir collectif. 
Une autre dissolution provient de l'opalescence inhérente à l'écriture littéraire, ses images, reflets et ombres, ses nombreuses constellations. Ainsi les sens se multiplient-ils pour, bientôt, se désagréger dans des cercles et des rifains toujours s'élargissant.

Mais nos dissolutions se poursuivent.

Le papier sur lequel les mots sont inscrits, est coupé aux ciseaux, scanné, sauvegardé, édité, modifié, digitalisé. Au cours de ce processus, le papier a disparu.

Les mots devenus images ont ensuite été étalés, superposés, brouillés ou fondus les uns dans les autres jusqu'à ce qu'ils deviennent tellement insaisissables que nous ne pouvions plus les distinguer. La vidéo qui en résulte, a pour dessein d'accomplir un geste fluide d'effacement.

Finalement, les effets sonores accompagnateurs - Ominous dance floor, Circuit dialog, Gorai atmosphere, Samoa sweep et Serena swirl - ont été choisis parce qu'ils font obstacle à cette malléabilité. Avec l'intention d'éviter toute impression de spiritualité, nous avons sélectionné des sons caverneux qui perturbaient et faisaient taire le flux linguistique et visuel. Nos mots sont à présents engloutis dans l'abysse de ces accents immergés - leur dissolution finale.

Participating writers / Auteurs ayant participé:

Kate Mercer; Rachael Campbell; Megan Franklin; Elizabeth Ratko; Colleen M.; Jessica McQuiggin; Carla Cattafi; Jackie Woroniuk; Samantha Schmid; Stephanie Bogucki; Kate Hale; Ana Haljkevic; Ambika Sangaran; Catherine Parayre; Rodrigo León Cordero; Veronica Mercuri; Stéphanie Kaboré; Arthur Sarmento Furtado; Ramon Augusto Porto Beranger Vieira; Débora Boccolini Paiva; Axelle Mroz; Su Mi Lee; Joungzk Park; Brandon Garib; Lyne-Duchesse Iraduhaye ; Waleed AlBuri; Abdulmajeed Almaimani; Abdullah Aljasser; Abddulkader Alomari; Jody Wen; Le Wang; Zhao Ying; Yue Deng; Nabilah Alhaddad; Omar Sulaiman Alajaimi; Yinfeng Lu; Jin Xiufeng; Zhao Yang; Siming Chen; Wang Lu; Berta Ferenc A'ron; Wang Fan; Han Xu; Qili Luo; Faisal Suliman Aldemaiji; Linlin Zhang; Edison Chow; Anas Almaghrabi; John Shi; Jiayu Wang (Jady); Lisette Costanzo; Simon Yin; Jacob Braun; Nafée Faïgou; Mathew Piccirillo; Milke Frydryk; Sam Franchi.

Thanks go to Duncan MacDonald, Gregory Betts, Adam Dickinson, and Paul Savoie. Remerciements à Duncan MacDonald, Gregory Betts, Adam Dickinson et Paul Savoie. 